\title{
The Thickness and Stretch Dependence of the Electrical Breakdown Strength of an Acrylic Dielectric Elastomer
}

\section{Citation}

Huang, Jiangshui, Samuel Shian, Roger M. Diebold, Zhigang Suo, and David R. Clarke. 2012. The thickness and stretch dependence of the electrical breakdown strength of an acrylic dielectric elastomer. Applied Physics Letters 101:122905.

\section{Published Version}

doi:10.1063/1.4754549

\section{Permanent link}

http://nrs.harvard.edu/urn-3:HUL.InstRepos:9887625

\section{Terms of Use}

This article was downloaded from Harvard University's DASH repository, and is made available under the terms and conditions applicable to Open Access Policy Articles, as set forth at http:// nrs.harvard.edu/urn-3:HUL.InstRepos:dash.current.terms-of-use\#OAP

\section{Share Your Story}

The Harvard community has made this article openly available.

Please share how this access benefits you. Submit a story.

\section{Accessibility}




\title{
THE THICKNESS AND STRETCH DEPENDENCE OF THE ELECTRICAL BREAKDOWN STRENGTH OF AN ACRYLIC DIELECTRIC ELASTOMER
}

\author{
Jiangshui Huang ${ }^{1}$, Samuel Shian ${ }^{1}$, Roger M. Diebold ${ }^{1,2}$, Zhigang Suo ${ }^{1, a}$ and David R. Clarke ${ }^{1, b}$ \\ ${ }^{1}$ Harvard School of Engineering and Applied Sciences, \\ Harvard University, Cambridge, MA 02138 \\ ${ }^{2}$ Materials Department, University of California, \\ Santa Barbara, Santa Barbara, CA 93106
}

\begin{abstract}
The performance of dielectric elastomer actuators is limited by electrical breakdown. Attempts to measure this are confounded by the voltage-induced thinning of the elastomer. A test configuration is introduced that avoids this problem: A thin sheet of elastomer is stretched, crossed-wire electrodes attached, and then embedded in a stiff polymer. The applied electric field at breakdown $E_{B}$ is found to depend on both the deformed thickness, $h$, and the stretch applied, $\lambda$. For the acrylic elastomer investigated, the breakdown field scales as $E_{B}=51 h^{-0.25} \lambda^{0.63}$. The test configuration allows multiple individual tests to be made on the same sheet of elastomer.
\end{abstract}

Keywords: Dielectric elastomers, Dielectric strength, Electric breakdown, Dielectric thinning

Corresponding authors:

a) suo@seas.harvard.edu

b) clarke@seas.harvard.edu 
Thin dielectrics are widely used to provide electrical insulation for a wide variety of electronic and electrical devices, often integrated into the device structures themselves. The most notable examples of this are the nanometer-scale silica dielectric layer in CMOS devices and the silica-carbon- oxy-fluoride glasses used to insulate the metal interconnect structures distributing power and signals in silicon devices. Thicker dielectrics, such as sleeves and sheaths, are used to insulate wires and electrical cables; for instance, high-density polyethylene is usually the material of choice for high voltage electric power cables. Although these and other insulating dielectric materials are very different and the mechanisms of failure may not be the same, it has been known for many years that there is a general empirical relationship between the breakdown field, $E_{B}$, and the thickness, $h$, of the dielectric, $E_{B} \propto h^{-n} \cdot{ }^{1-9}$ This relationship fits data for amorphous solids, such as silica glass and polymers, semi-crystalline polymers as well as a wide range of crystalline ceramics, including polycrystalline and single crystal alumina ${ }^{10}$ although the values of $n$ are not unique. The majority of insulating solids are stiff and do not deform appreciably in response to the Coulomb attraction force between oppositely charged electrodes when an electric field is applied across them. This absence of deformation is not the case for soft elastomers and indeed their change in thickness with an applied voltage has been exploited to create electric field-induced actuators. ${ }^{11-22}$ Very large actuation strains can be achieved using soft elastomer dielectrics but they must first be biaxially stretched. It is now understood that pre-straining is necessary to forestall an electromechanical instability mode associated with dielectric thinning identified by Stark and Garton. ${ }^{23}$ They showed that as the voltage across 
an elastomer is increased, it thins and, in doing so, the electric field increases and that this coupled positive feedback process continues until the local breakdown field is attained. Prestretching stiffens the dielectric and may even remove the electromechanical instability. ${ }^{14}$ Prestretching also increases the breakdown field. ${ }^{24-26}$ For instance, for the most widely studied dielectric elastomer, the acrylic elastomer produced by $3 \mathrm{M}$ corporation, VHB, it has been reported that the breakdown electric field increases from 18 to $218 \mathrm{MV} / \mathrm{m}$ when equi-biaxially stretched up to 6 times. $^{25}$ Since the maximum actuation strains for soft dielectric actuators are limited by electrical breakdown, the effect of electrical breakdown on thickness is of particular practical importance. Furthermore, from a practical perspective, the thinning in response to an applied voltage poses a challenge to how to measure the electrical breakdown field.

The traditional method of making electrical breakdown measurements is to use large area electrodes, much larger than the dielectric thickness, and ramp up the voltage until breakdown occurs. ${ }^{2,3}$ This is the method that has previously been used to evaluate the breakdown field of elastomers. ${ }^{24-26}$ The other method consists of a flat electrode on one side of the dielectric and a curved electrode on the other, with a curvature typically large compared to the dielectric thickness. ${ }^{27}$ In both test geometries, the electrodes are generally free to displace towards one another when the voltage is increased unless the electrodes are very stiff. Indeed, in some cases such as the curved electrode configuration test, the curved electrode is pressed into contact with the dielectric to ensure good electrical contact.

In this contribution we describe an alternative testing geometry that prevents displacements of the electrodes as the voltage is increased and use it to determine 
breakdown data for the commonly investigated dielectric acrylic elastomer, VHB. Based on preliminary experiments, our experiments are designed to separate the effects of thickness and pre-stretch. Elastomer sheets of two starting thicknesses were investigated, one $0.5 \mathrm{~mm}$ in thickness (VHB 4905) and the other $1.0 \mathrm{~mm}$ thick (VHB 4910). (Both sheets have the same polymer composition, the last two numbers in the notation refer to the sheet thickness). A bi-layer, also $1 \mathrm{~mm}$ thick, was produced by pressing two sheets of VHB 4905 together. Stretch is defined as the ratio of the length after stretching to the original length of the elastomer. The stretches were all equi-biaxial and are denoted by $\lambda$ (Fig.1). The elastomer is taken to be incompressible, so that its deformed thickness is related to its stretch by the relationship $h=H / \lambda^{2}$, where $h$ is the deformed thickness of the sheet and $H$ is the initial thickness.

Our electrical test arrangement is shown in figure 1 . It consists of an elastomer sheet with an array of parallel cylindrical wires on either side, all embedded by casting a stiff polymer around them. There are two main features of the test configuration: the elastomer is prevented from thinning and changing dimensions by the stiff polymer block in which the elastomer is embedded and the wires encapsulated in the stiff polymer on either side of the elastomer are arranged at right angles to one another. A further feature of the test arrangement is that the elastomer sheet can be pre-stretched biaxially prior to the placement of the wire electrodes and subsequent embedding of the elastomer. The polymer blocks on either side of the elastomer sheet were made from a two component polyurethane mixture (Smooth Cast 300, Smooth-On) that, once cross-linking had initiated, was cast around the stretched elastomer to form a stiff plastic layer about $3 \mathrm{~mm}$ in thickness 
after fully curing. The cast polymer is not only much thicker than the VHB elastomer but also orders of magnitude stiffer (Young's modulus=1.0 GPa versus 1.8 MPa of the VHB according to $3 \mathrm{M}$ datasheet). After allowing the polymer to fully cure, it was removed from the mold and electrical connection was made to the individual wires. The electrodes consisted of high purity (99.99\%) copper wires, with a diameter of $18 \mu \mathrm{m}$ (Semiconductor Packaging Materials). The array of parallel, crossed wires facilitates making a series of breakdown measurements all at the same elastomer thickness and stretch and on the same sample. The arrangement allows for a large number of individual tests to be made, and the average breakdown voltages for each sheet thickness and stretch were determined from no fewer than 24 tests on each sample.

All the measurements were made at room temperature and the test consisted of increasing the applied voltage (Gamma High voltage Research, model number ES40P-5W-DAM) at a fixed ramp rate of $50 \mathrm{~V} / \mathrm{s}$ until electric breakdown occurred at a voltage, $\Phi_{B}$. The breakdown criterion was when the current exceeded the 125 micro-amp pre-set of the voltage source. This was usually accompanied by a spark, apparent visually in many instances, and a "popping" sound. The breakdown paths were conducting afterwards suggesting that thermal breakdown and subsequent carburization occurred before the voltage supply automatically cut-off. The values of the breakdown fields reported here are the engineering breakdown fields, namely the voltage divided by the deformed dielectric thickness:

$$
E_{B}=\Phi_{B} \lambda^{2} / H
$$

The test configuration was chosen for a number of reasons. It suppresses deformation of 
the elastomer under voltage and localizes the electric field more effectively than small pads, decreasing the volume of the dielectric subject to high fields. It also allows a large number of test sites to be addressed without the necessity of precisely having to align small pads and make separate connections to them.

Our measurements show that the average breakdown voltage decreases monotonically with increasing stretch (Fig. 2a), consistent with previous measurements of the same elastomer ${ }^{24-26}$ but our numerical values are almost twice as large. We believe that the larger breakdown fields are a direct consequence of the smaller volume of material under electric field in our cross-cylinder test configuration. The existence of a size effect is consistent with previous studies of the breakdown field of several materials, including one on elastomers. ${ }^{28,}$ ${ }^{29}$ Amongst the clearest studies of electrode size effects is the recent study of hafnia and silica films which also show that the smaller the contact area, the larger is the breakdown field. ${ }^{30}$ We also observe a thickness dependence of the electric breakdown field (Fig. 2b) but this is less marked than has previously been reported. By comparing, the breakdown fields in figure 2 (b) for the elastomer sheets of different initial thicknesses it was found that the breakdown electric fields do not collapse onto a single curve. From this we conclude that the breakdown field depends not only on the thickness but also on the stretch. This conclusion is substantiated by comparing the electric breakdown field as a function of stretch for the two elastomers of different thickness and a bilayer VHB4905 as is shown in figure 2(c).

Empirically, we can write that the breakdown field depends on both the deformed thickness and the stretch: $E_{B}=C h^{-x} \lambda^{y} \quad$ where $C$ is a constant and $x$ and $y$ are exponents to be determined. All the breakdown data for the elastomer with different thicknesses and 
values of pre-stretch collapse onto a single scaling as shown in figure 2 (d), corresponding to $E_{B}=51 h^{-0.25 \pm 0.01} \lambda^{0.63 \pm 0.03}$, where the field is in units of $\mathrm{MV} / \mathrm{m}$ and the thickness is in $\mathrm{mm}$. In terms of the initial thickness of the dielectric, the breakdown field can alternatively be expressed as $E_{B}=51 H^{-0.25} \lambda^{1.13}$. The stretch exponent, 1.13 , is very similar to that, 1.12 , based on fitting to Kofod's data ${ }^{25}$ (Fig.2c) although the pre-factor is somewhat larger, suggesting that nevertheless the same physical processes are involved.

The observed dependence of the breakdown field on the elastomer pre-stretch suggests a number of possibilities. One is that the dielectric constant, $\varepsilon$, of the elastomer varies with deformation and depends on the pre-stretch. Unfortunately, there is conflicting information in the literature as to whether the dielectric constant or the refractive of the VHB materials depends on pre-stretching. Kofod et al. reported that the dielectric constant only slightly dropped with increase of the pre-stretch up to 6 but there is another report that the dielectric constant decreases from its nominal value of 4.7 to 2.6 at a stretch of $5 .{ }^{25,31} \mathrm{~A}$ much smaller reduction, up to $15 \%$, has been reported when a pre-strain is applied. ${ }^{32,33}$ As it is expected that breakdown occurs when a critical value of the electric field inside the dielectric , $E_{B}^{\mathrm{int}}$, is attained and that is related to the applied field , $E_{\text {applied }}$, by, $E_{B}^{\text {int }}=E_{\text {applied }} / \varepsilon$, decreasing the dielectric constant by stretching would decrease the applied breakdown field rather than increase it as is observed. Another possibility is that in stretching the elastomer, the vibrational amplitudes of the individual molecules are reduced and consequently the activation volume is decreased through which mobile charges can migrate under field. Such an activated state description applies to the breakdown of many amorphous polymers, such as high-density polyethylene and polypropylene. ${ }^{3}$ However, this 
form of thermally activated breakdown is usually applicable to breakdown under sustained field conditions rather than short ramping conditions. Finally, we are not in a position to evaluate a third possible breakdown model associated with shape changes due to cavities, such as bubbles, under field although we believe that our test configuration avoided the entrapment of air bubbles. As long ago as 1964, Garton and Krasucki ${ }^{4,34}$ showed that electrical breakdown in an insulating viscous liquid could occur when an air bubble is entrained. Under the influence of the field, the bubble changes shape, elongating in the direction of the field, until breakdown occurred. Essentially, the same mechanism has recently been invoked in soft elastomers containing bubbles by Wang et al. ${ }^{27}$ However, knowing of this work, we took great care to avoid the entrapment of any bubbles. Furthermore, the probability of having a small bubble in each test volume in the crossed cylinder geometry seems highly unlikely. One would also expect that if this were a viable mechanism, bubbles at the interface between the two $0.5 \mathrm{~mm}$ sheets of VHB 4905 would have grown and the breakdown fields would be consistently smaller but this is not the case.

In summary, by using a constrained test geometry that prevents thinning in response to an applied geometry and possible electromechanical instability, we have demonstrated that the electric breakdown field of an elastomer sheet commonly used in dielectric actuators depends on both thickness and the biaxial pre-stretch. The breakdown field is found to scale according to simple power law $E_{B}=51 h^{-0.25} \lambda^{0.63}$ over the range of thickness and mechanical stretching studied. More detailed studies are necessary to investigate the scaling over a greater range of thicknesses and stretches as well as how stretch affects physical properties including dielectric constant and optical properties. Measurements of the 
sustained voltage, time, and temperature dependence are also needed to assess voltage creep.

\section{Acknowledgements}

Our research was supported by both the Harvard MRSEC program of the National Science Foundation under Award Number DMR-0820484 and by the Gordon McKay endowment. 


\section{References}

1. A. E. W. Austen and S. Whitehead, Proc. R. Soc. Lond. A 1940 176, 33-50.

2. S. Whitehead, "Dielectric breakdown of solids", Clarendon Press, Oxford, 1953.

3. L. A. Dissado, J. C. Fothergill, "Electrical Degradation and Breakdown in Polymers", Peter Peregrinus Ltd., London, United Kingdom, 1992.

4. K.C. Kao, "Dielectric Phenomena in Solids", Elsevier Academic Press, San Diego, California, USA, 2004.

5. F. Forlani, N. Minnaja, Phys. Stat. Sol. 4, 311 (1964).

6. V.K. Agarwal, V.K. Srivastava, Thin Solid Films 8, 377(1971).

7. V.K. Agarwal, V.K. Srivastava, Solid State Commun. 12, 829 (1973).

8. B. Helgee, P. Bjellheim, IEEE Trans. Electr. In. 26, 1147 (1991).

9. H.K. Kim, F.G. Shi, IEEE T. Dielect. El. In. 8, 248 (2001).

10. M. Yoshimura, H. K. Bowen, J. Am. Ceram. Soc. 64, 404 (1981)

11. R. Pelrine, R. Kornbluh, Q. Pei., J. Joseph, Science 287, 836 (2000).

12. J. Huang, T. Li, C. C. Foo, J. Zhu, D. R. Clarke, and Z.G. Suo, Appl. Phys. Lett. 100, 041911 (2012).

13. J. Huang, T. Lu, J. Zhu, D. R. Clarke, and Z.G. Suo, Appl. Phys. Lett., 100, 211901 (2012).

14. T. Lu, J. Huang, C. Jordi, G. Kovacs, R. Huang, D. R. Clarke and Zhigang Suo, Soft Matter, 8, 6167-6173 (2012)

15. G. Kovacs, L. During, S. Michel, G. Terrasi, Sensors Actuators A 155, 299 (2009).

16. C. Keplinger, M. Kaltenbrunner, N. Arnold, and S. Bauer, Proc. Nat. Acad. Sci. 107, 4505 (2010).

17. F. Carpi, G. Frediani, S. Turco, and D. De Rossi, Adv. Funct. Mater. 21, 4152 (2011).

18. I. A. Anderson, T. C. H. Tse, T. Inamura, B. M. O’Brien, T. McKay, T. Gisby, Appl. Phys. Lett. 98, 123704 (2011).

19. B. M. O’ Brien, T. G. McKay, T. A. Gisby, I. A. Anderson, Appl. Phys. Lett. 100, 074108 (2012).

20. A. T. Conn, J. Rossiter, Smart Mater. Struct. 21, 035012 (2012).

21. A. Cheng, "ViviTouchTM Offers a New Sensory Dimension to Mobile Gaming", WW-EAP Newsletter, 13, 2 (2011).

22. P. Chouinard and J. Plante, IEEE-ASME T. Mech. 17, 857(2012).

23. K. H. Stark, C. G. Garton, Nature, 176, 1225 (1955)

24. J. S. Plante, S. Dubowsky, J. Solids Struct. 43, 7727(2006).

25. G. Kofod, P. Sommer-Larsen, R. Kornbluh, R. Pelrine, J. Intell. Mater. Syst. Struct. 14, 787 (2003).

26. C. Jordi, A. Schmidt, G. Kovas, S. Michel, P. Ermanni, Smart Mater. Struct. 2o, 075003 (2011).

27. Q. Wang, M. Tahir, L. Zhang, X. Zhao, Soft Matter 7, 6583(2011).

28. S.Cygan, J.R. Laghari, IEEE Trans. Electr. In. EI-22, 835 (1987). 
29. S. Diaham, S. Zelmat, M.L. Locatelli, S. Dinulescu, M. Decup, T. Lebey, IEEE T. Dielect. El. In. 17, 18(2010).

30. C. Sire, S. Blonkowski, M.J. Gordon, T. Baron, APL 91, 242905(2007).

31. M. Wissler, E. Mazzab, Sens. Actuators A 138, 384 (2007).

32. T.G. Mckay, E. Calius, I.A. Anderson, Proc. of SPIE 7287, 72870 P (2009).

33. C. Jean-Mistral, A. Sylvestre, S. Basrour, J-J Chaillout, Smart Mater. Struct. 19, 075019 (2010).

34. C. G. Garton; Z. Krasucki, Proc. R. Soc. London, Ser. A 280, 211 (1964). 


\section{Figure}

(a)

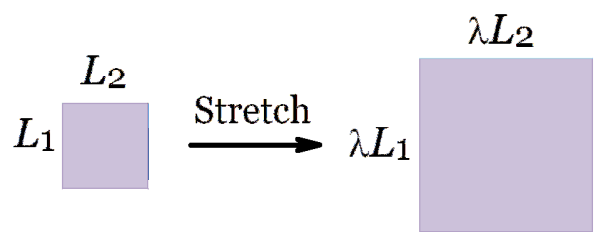

(b)

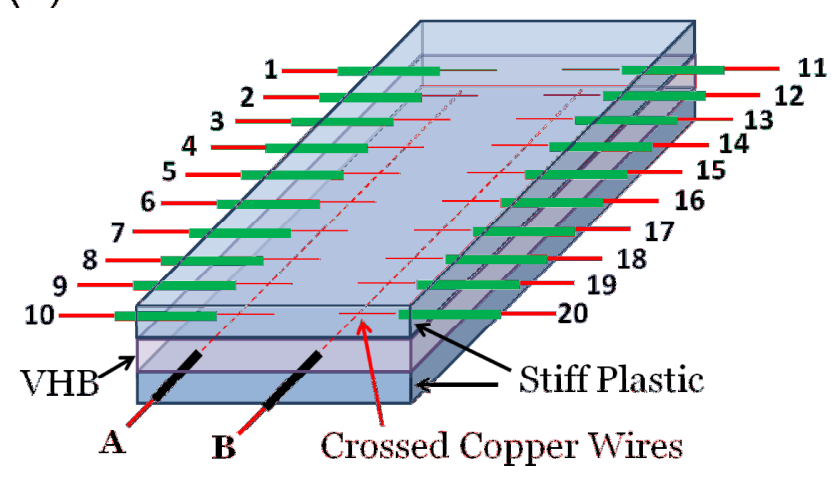

FIG.1 (Color online) (a) An elastomer sheet is stretched biaxially by an amount denoted as the stretch ratio $\lambda$; (b) Electrical test arrangement consists of a prestretched elastomer sheet with an array of parallel cylindrical wires on either side, all embedded by casting a stiff polymer around them. The polymer blocks on either side of the elastomer sheet were made from a two component polyurethane mixture that, once cross-linking had initiated, was cast around the stretched elastomer to form a stiff plastic layer about $3 \mathrm{~mm}$ in thickness after fully curing. The electrodes consisted of high purity (99.99\%) copper wires with a diameter of $18 \mu \mathrm{m}$ encapsulated in the stiff polymer on either side of the elastomer are arranged at right angles to one another. Electrical connection was made to the individual wires, allowing for a large number of individual tests to be made on the same sheet. For example, 20 tests can be made in the configuration shown in the schematic. 

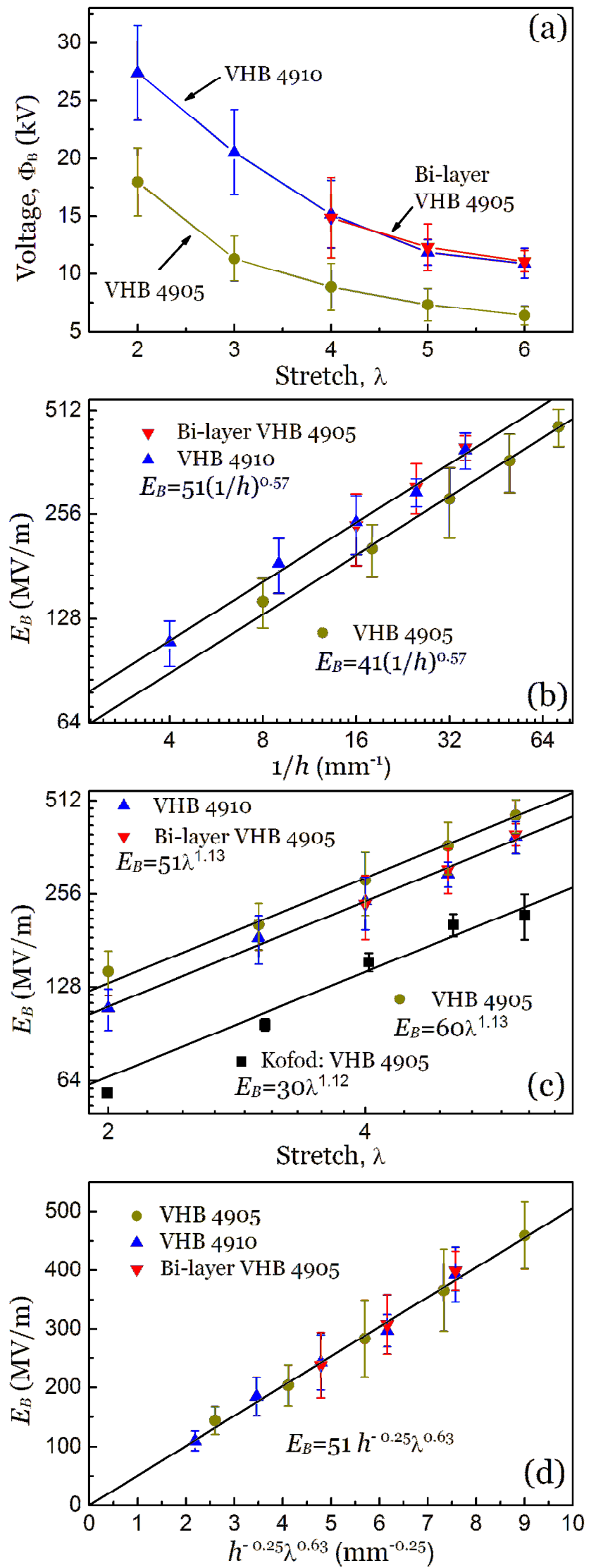

FIG.2 (Color online) (a) For each initial dielectric thickness, the breakdown voltages decrease monotonically with increase of stretch. (b) Breakdown electric field as a function of thickness for VHB 4905, two-layer VHB 4905 and VHB 4910 stretched to the values 
indicated. The lines through the data correspond to a power law dependence on $(1 / h)$ with an exponent of 0.57. Data for VHB 4910 and two-layer VHB 4905 are fitted to same line. The vertical displacement between the two lines indicates the dielectric strength also depends on the stretch. (c) Breakdown electric field as a function of stretch. The lines through the data correspond to a power law dependence on $\lambda$ with an exponent of 1.13. Again the difference between the two lines indicates the dielectric strength should depend on the thickness. Data from the work of Kofod are also included and an exponent of 1.12 is obtained by fitting. (d) Experimentally determined scaling between the breakdown electric field and the parameter $\left(h^{-0.25} \lambda^{0.63}\right)$. 